\title{
Symmetries of Gromov-Witten Invariants
}

\author{
Alexander Postnikov \\ Department of Mathematics, University of California, Berkeley, CA 94720 \\ apost@math . berkeley . edu
}

September 17, 2000

ก

N

\begin{abstract}
The group $(\mathbb{Z} / n \mathbb{Z})^{2}$ is shown to act on the Gromov-Witten invariants of the complex flag manifold. We also deduce several corollaries of this result.
\end{abstract}

\section{Introduction}

The aim of this paper is to present certain symmetry properties of the GromovWitten invariants for type $A$ complex flag manifolds.

Recall that the cohomology ring of the complex flag manifold $F l_{n}$ has an additive basis of Schubert classes $\sigma_{w}$, which are indexed by permutations $w$ in the symmetric group $S_{n}$. For permutations $u, v, w \in S_{n}$, the Schubert number $c_{u, v, w}$ is the structure constant of the cohomology ring in the basis of Schubert classes:

$$
\sigma_{u} \cdot \sigma_{v}=\sum_{w \in S_{n}} c_{u, v, w} \sigma_{w_{\mathrm{o}} w}
$$

where $w_{\mathrm{o}}$ is the longest permutation in $S_{n}$. Equivalently,

$$
c_{u, v, w}=\int \sigma_{u} \cdot \sigma_{u} \cdot \sigma_{w}
$$

is the intersection number of Schubert varieties. Thus these numbers are nonnegative integers symmetric in $u, v$, and $w$. They generalize the famous LittlewoodRichardson coefficients. If $\ell(u)+\ell(v)+\ell(w) \neq \frac{n(n-1)}{2}$ then the Schubert number $c_{u, v, w}$ is zero for an obvious degree reason.

A long standing open problem is to find an algebraic or combinatorial construction for the coefficients $c_{u, v, w}$ that would imply their nonnegativity. A possible approach to this problem could be in its generalization to the quantum cohomology ring of the flag manifold $F l_{n}$. The structure constants of this ring are certain polynomials whose coefficients are the Gromov-Witten invariants $\left\langle\sigma_{u}, \sigma_{v}, \sigma_{w}\right\rangle_{\left(d_{1}, \ldots, d_{n-1}\right)}$ The Schubert number $c_{u, v, w}$ is a special case of the Gromov-Witten invariants: $c_{u, v, w}=\left\langle\sigma_{u}, \sigma_{v}, \sigma_{w}\right\rangle_{(0, \ldots, 0)}$. These invariants are 
defined as numbers of certain rational curves in $F l_{n}$. The geometric definition of the Gromov-Witten invariants implies their nonnegativity.

In this paper we establish cyclic symmetries of the Gromov-Witten invariants that could not be detected in their full generality on the "classical" level of the Schubert numbers $c_{u, v, w}$. Several related results for the $c_{u, v, w}$ when $u$ is a Grassmannian permutation were, however, found by Bergeron and Sottile, see [2, Theorems 1.3.4, 1.3.4]. In case of the Gromov-Witten invariants we do not need to restrict the rule to Grassmannian permutations. Similar symmetries of the Gromov-Witten invariants for Grassmannian varieties were found in [i].

\section{Gromov-Witten invariants}

Let $F l_{n}$ denote the manifold of complete flags of subspaces in the complex $n$ dimensional linear space $\mathbb{C}^{n}$. One can also define the flag manifold as $F l_{n}=$ $G L_{n}(\mathbb{C}) / B$, where $B$ is the Borel subgroup of upper triangular matrices in the general linear group. The flag manifold is a compact smooth complex manifold. For a permutation $w \in S_{n}$, the Schubert variety $X_{w}$ is the closure of the Schubert cell $B_{-} w B / B$ in $F l_{n}$, where $B_{-}$is the subgroup of lower triangular matrices and $w$ is viewed as a permutation matrix in $G L_{n}$. The Schubert classes $\sigma_{w} \in$ $\mathrm{H}^{*}\left(F l_{n}, \mathbb{Z}\right)$, indexed by permutations $w \in S_{n}$, are defined as the Poincaré duals of the homology classes $\left[X_{w}\right]$ of Schubert manifolds. They form an additive $\mathbb{Z}$-basis of the cohomology ring $\mathrm{H}^{*}\left(F l_{n}, \mathbb{Z}\right)$. Moreover, $\sigma_{w} \in \mathrm{H}^{2 l}\left(F l_{n}, \mathbb{Z}\right)$, where $l=\ell(w)$ is the length of permutation $w$, i.e., its number of inversions.

Recently, attention has been drawn to the (small) quantum cohomology ring $\mathrm{QH}^{*}\left(F l_{n}, \mathbb{Z}\right)$ of the flag manifold. The definition of quantum cohomology can be found, for example, in [5]. Here we briefly outline several notions and results.

As a vector space, the quantum cohomology of $F l_{n}$ is the usual cohomology tensored with the polynomial ring in $n-1$ variables:

$$
\mathrm{QH}^{*}\left(F l_{n}, \mathbb{Z}\right) \cong \mathrm{H}^{*}\left(F l_{n}, \mathbb{Z}\right) \otimes \mathbb{Z}\left[q_{1}, \ldots, q_{n-1}\right] .
$$

The Schubert classes $\sigma_{w}$, thus, form a $\mathbb{Z}\left[q_{1}, \ldots, q_{n-1}\right]$-basis of the quantum cohomology ring.

The multiplication in $\mathrm{QH}^{*}\left(F l_{n}, \mathbb{Z}\right)$ (quantum product) is a commutative $\mathbb{Z}\left[q_{1}, \ldots, q_{n-1}\right]$-linear operation. It is therefore sufficient to specify the quantum product of any two Schubert classes. To avoid confusion with the multiplication in the usual cohomology ring, we will use "*" to denote the quantum product. The quantum product $\sigma_{u} * \sigma_{v}$ of two Schubert classes can be expressed in the basis of the Schubert classes as

$$
\sigma_{u} * \sigma_{v}=\sum_{w \in S_{n}} C_{u, v, w} \sigma_{w_{\mathrm{o}} w},
$$

where $C_{u, v, w} \in \mathbb{Z}\left[q_{1}, \ldots, q_{n-1}\right]$ and $w_{\mathrm{o}}=\left(\begin{array}{cccc}1 & 2 & \cdots & n \\ n & n-1 & \cdots & 1\end{array}\right)$ is the longest permutation in $S_{n}$. 
The coefficient of $q_{1}^{d_{1}} \cdots q_{n-1}^{d_{n-1}}$ in the polynomial $C_{u, v, w}$ is the Gromov-Witten invariant $\left\langle\sigma_{u}, \sigma_{v}, \sigma_{w}\right\rangle_{\left(d_{1}, \ldots, d_{n-1}\right)}$. The Gromov-Witten invariants are defined geometrically as numbers of certain rational curves in $F l_{n}$. (See [5] or [3] for details.) Let us summarize the main properties of these invariants. It will be more convenient for us to work with the polynomials $C_{u, v, w}$.

1. (Nonnegativity) All coefficients of the $C_{u, v, w}$ are nonnegative integers.

2. ( $S_{3}$-symmetry) The polynomials $C_{u, v, w}$ are invariant with respect to permuting $u$, $v$, and $w$.

3. (Degree condition) The polynomial $C_{u, v, w}$ is a homogeneous polynomial of degree $\frac{1}{2}\left(\ell(u)+\ell(v)+\ell(w)-\frac{n(n-1)}{2}\right)$.

4. (Classical limit) The Schubert number $c_{u, v, w}$ is the constant term of the polynomial $C_{u, v, w}$.

5. (Associativity) The operation "*” defined by (2) via the polynomials $C_{u, v, w}$ is associative.

The first four properties are clear from geometric definitions. It was conjectured in [3] that nonnegativity, associativity, degree condition, and classical limit condition uniquely determine the Gromov-Witten invariants.

The conditions $\mathbf{3}$ and $\mathbf{4}$ immediately imply the following statement.

Proposition 1 We have

$$
C_{u, v, w}=\left\{\begin{array}{cl}
0 & \text { if } \ell(u)+\ell(v)+\ell(w)<\frac{n(n-1)}{2}, \\
0 & \text { if } \ell(u)+\ell(v)+\ell(w)-\frac{n(n-1)}{2} \text { is odd, } \\
c_{u, v, w} & \text { if } \ell(u)+\ell(v)+\ell(w)=\frac{n(n-1)}{2}, \\
? ? ? & \text { overwise. }
\end{array}\right.
$$

In [3] we gave a method for calculation of the Gromov-Witten invariants. Among several approaches presented in that paper, one is based on the quantum analogue of Monk's formula.

For $1 \leq i<j \leq n$, let $s_{i j}$ be the transposition in $S_{n}$ that permutes $i$ and $j$. Then $s_{i}=s_{i i+1}$ is an adjacent transposition. Also, let $q_{i j}$ be a shorthand for the product $q_{i} q_{i+1} \cdots q_{j-1}$.

Proposition 2 [3, Theorem 1.3] (quantum Monk's formula) For $w \in S_{n}$ and $1 \leq k<n$, the quantum product of the Schubert classes $\sigma_{s_{k}}$ and $\sigma_{w}$ is given by

$$
\sigma_{s_{k}} * \sigma_{w}=\sum \sigma_{w s_{a b}}+\sum q_{c d} \sigma_{w s_{c d}}
$$

where the first sum is over all transpositions $s_{a b}$ such that $a \leq k<b$ and $\ell\left(w s_{a b}\right)=\ell(w)+1$, and the second sum is over all transpositions $s_{c d}$ such that $c \leq k<d$ and $\ell\left(w s_{c d}\right)=\ell(w)-\ell\left(s_{c d}\right)=\ell(w)-2(d-c)+1$. 
Remark 3 The two-dimensional Schubert classes $\sigma_{s_{k}}$ generate the quantum cohomology ring. Thus formula (3) uniquely determines the multiplicative structure of $\mathrm{QH}^{*}\left(F l_{n}, \mathbb{Z}\right)$ and, therefore, the Gromov-Witten invariants.

\section{Cyclic symmetry}

Let $o=(1,2, \ldots, n)$ be the cyclic permutation in $S_{n}$ given by

$$
o(i)=i+1, \text { for } i=1, \ldots, n-1, \quad o(n)=1 .
$$

Recall that $q_{i j}=q_{i} q_{i+1} \cdots q_{j-1}$ for $i<j$. We also define $q_{i j}=q_{j i}^{-1}$ for $i>j$ and $q_{i i}=1$.

Theorem 4 For any $u, v, w \in S_{n}$ we have

$$
C_{u, v, w}=q_{i j} C_{u, o^{-1} v, o w},
$$

where $i=v^{-1}(1)$ and $j=w^{-1}(n)$.

The $S_{3}$-invariance of the $C_{u, v, w}$ under permuting $u, v$, and $w$ implies a more general statement.

For $w \in S_{n}$ and $1 \leq a \leq n$, define the following Laurent monomials in the $q_{i}$

$$
Q_{w, a}=\prod_{i: w(i) \geq n-a+1} q_{1 i}, \quad Q_{w,-a}=\prod_{j: w(j) \leq a}\left(q_{1 j}\right)^{-1},
$$

and let $Q_{w, 0}=1$.

Corollary 5 For any $u, v, w \in S_{n}$ and $-n \leq a, b, c \leq n$ such that $a+b+c=0$, we have

$$
C_{u, v, w}=Q_{u, a} Q_{v, b} Q_{w, c} C_{o^{a} u, o^{b} v, o^{c} w} .
$$

In many cases Corollary 同 and Proposition 1 allow us to reduce the polynomials $C_{u, v, w}$ to the Schubert numbers $c_{u, v, w}$ :

Corollary 6 For $u, v, w \in S_{n}$, let us find a triple $-n \leq a, b, c \leq n, a+b+c=0$, for which the expression

$$
\ell_{a, b, c}=\ell\left(o^{a} u\right)+\ell\left(o^{b} v\right)+\ell\left(o^{c} w\right)
$$

is as small as possible. If $\ell_{a, b, c}<\frac{n(n-1)}{2}$ then $C_{u, v, w}=0$. If $\ell_{a, b, c}=\frac{n(n-1)}{2}$ then $C_{u, v, w}=Q_{u, a} Q_{v, b} Q_{w, c} c_{o^{a} u, o^{b} v, o^{c} w}$.

Remark 7 (Reduction of Gromov-Witten invariants) The Gromov-Witten invariants have the following stability property. If $u, v, w \in S_{n}$ are three permutations such that $u(n)=v(n)=n$ and $w(n)=1$ then $C_{u, v, w}=C_{u^{\prime}, v^{\prime}, w^{\prime}}$, where 
$u^{\prime}, v^{\prime}, w^{\prime} \in S_{n-1}$ are permutations obtained from $u, v, w$ by removing the last entry (and subtracting 1 from all entries of $w$ ).

For a triple of permutation $u, v, w \in S_{n}$ such that $u(n)+v(n)+w(n) \equiv 1$ $(\bmod n)$, we can use the relation (5) to transform the triple to the above case when we can use the stability property. This shows that $1 / n$ of all GromovWitten invariants for $F l_{n}$ can be reduced to the Gromov-Witten invariants of $F l_{n-1}$. Analogously, we can reduce the problem to a lower level for a triple of permutations $u, v, w \in S_{n}$ such that $u(1)+v(1)+w(1) \equiv 2 \quad(\bmod n)$.

Remark 8 (New rules for multiplication of Schubert classes) Suppose that a rule is know for the quantum multiplication of an arbitrary Schubert class by certain Schubert class $\sigma_{u}$. Theorem 4 immediately produces a new rule for the quantum multiplication by $\sigma_{o^{a} u}$, where $a \in \mathbb{Z}$. For example, we get for free a rule for $\sigma_{o^{a}} * \sigma_{w}$. Quantum Monk's formula (3) can be extended to a rule for $\sigma_{o^{a} s_{k}} * \sigma_{w}$. More generally, quantum Pieri's formula [6, Corollary 4.3] extends to an explicit rule for $\sigma_{o^{a} u} * \sigma_{w}$, where $u$ is a permutation of the form $u=s_{k} s_{k+1} \cdots s_{k+l}$ or $u=s_{k} s_{k-1} \cdots s_{k-l}$.

\section{Twisted cyclic shift}

Let $T_{i j}, 1 \leq i<j \leq n$, be the $\mathbb{Z}\left[q_{1}, \ldots, q_{n-1}\right]$-linear operators that act on the quantum cohomology ring $\mathrm{QH}^{*}\left(F l_{n}, \mathbb{Z}\right)$ by

$$
T_{i j}: \sigma_{w} \longmapsto\left\{\begin{array}{cl}
\sigma_{w s_{i j}} & \text { if } \ell\left(w s_{i j}\right)=\ell(w)+1, \\
q_{i j} \sigma_{w s_{i j}} & \text { if } \ell\left(w s_{i j}\right)=\ell(w)-2(j-i)+1, \\
0 & \text { otherwise. }
\end{array}\right.
$$

Then quantum Monk's formula (3) can be written as:

$$
\sigma_{s_{k}} * \sigma_{w}=\sum_{i \leq k<j} T_{i j}\left(\sigma_{w}\right) .
$$

The operators $T_{i j}$ satisfy certain simple quadratic relations. The formal algebra defined by these relations was studied in [4] and [6].

Let us also define the twisted cyclic shift operator $O$ that acts on the quantum cohomology ring $\mathrm{QH}^{*}\left(F l_{n}, \mathbb{Z}\right)$, linearly over $\mathbb{Z}\left[q_{1}, \ldots, q_{n-1}\right]$, by

$$
O: \sigma_{w} \longmapsto q^{(w)} \sigma_{o w},
$$

where $q^{(w)}=q_{r n}$ with $r=w^{-1}(n)$.

Proposition 9 For any $1 \leq i<j \leq n$, the operators $T_{i j}$ and $O$ commute:

$$
T_{i j} O=O T_{i j} .
$$

The following lemma clarifies the conditions in the right-hand side of (6). Its proof is a straightforward observation. 
Lemma 10 Let $w \in S_{n}$ and $1 \leq i<j \leq n$. Then

1. $\ell\left(w s_{i j}\right)=\ell(w)+1$ if and only if for all $i \leq k \leq j$ we have

$$
w(k) \geq w(j) \geq w(i) \quad \text { or } \quad w(j) \geq w(i) \geq w(k) ;
$$

2. $\ell\left(w s_{i j}\right)=\ell(w)-\ell\left(s_{i j}\right)=\ell(w)-2(j-i)+1$ if and only if for all $i \leq k \leq j$ we have

$$
w(i) \geq w(k) \geq w(j) .
$$

Proof of Proposition 9 - The crucial observation is that, for fixed $i \leq k \leq j$, the set of permutations $w$ such that

$$
w(k) \geq w(j) \geq w(i) \quad \text { or } \quad w(j) \geq w(i) \geq w(k) \quad \text { or } \quad w(i) \geq w(k) \geq w(j)
$$

is invariant under the left multiplications of $w$ by the cycle $o$. This fact, together with Lemma 10, implies that $\left(T_{i j} O\right)\left(\sigma_{w}\right)$ is nonzero if and only if $T_{i j}\left(\sigma_{w}\right)$ is nonzero. Assume that $T_{i j}\left(\sigma_{w}\right) \neq 0$ and consider three cases:

I. Neither $w(i)$ nor $w(j)$ is equal to $n$. Then either of the conditions in the right-hand side of (6) is satisfied for $w$ if and only if the same condition is satisfied for $o w$. Also $q^{(w)}=q^{\left(w s_{i j}\right)}$. Thus $\left(T_{i j} O\right)\left(\sigma_{w}\right)=\left(O T_{i j}\right)\left(\sigma_{w}\right)$.

II. We have $w(j)=n$. Then $w(i)<w(j)$ and $o w(i)>o w(j)$. Thus $\ell\left(w s_{i j}\right)=$ $\ell(w)+1$ and $\ell\left(o w s_{i j}\right)=\ell(o w)-\ell\left(s_{i j}\right)$. Thus $T_{i j}\left(\sigma_{w}\right)=\sigma_{w s_{i j}}$ and $T_{i j}\left(\sigma_{o w}\right)=$ $q_{i j} \sigma_{o w s_{i j}}$. Also we have $q^{(w)}=q_{j n}$ and $q^{\left(w s_{i j}\right)}=q_{i n}$. Therefore, $\left(T_{i j} O\right)\left(\sigma_{w}\right)=$ $q_{i j} q_{j n} \sigma_{o w s_{i j}}=q_{i n} \sigma_{o w s_{i j}}=\left(O T_{i j}\right)\left(\sigma_{w}\right)$.

III. We have $w(i)=n$. Then $w(i)>w(j)$ and $o w(i)<o w(j)$. Thus $\ell\left(w s_{i j}\right)=\ell(w)-\ell\left(s_{i j}\right)$ and $\ell\left(o w s_{i j}\right)=\ell(o w)+1$. Thus $T_{i j}\left(\sigma_{w}\right)=q_{i j} \sigma_{w s_{i j}}$ and $T_{i j}\left(\sigma_{o w}\right)=\sigma_{o w s_{i j}}$. Also we have $q^{(w)}=q_{i n}$ and $q^{\left(w s_{i j}\right)}=q_{j n}$. Therefore, $\left(T_{i j} O\right)\left(\sigma_{w}\right)=q_{i n} \sigma_{o w s_{i j}}=q_{i j} q_{j n} \sigma_{o w s_{i j}}=\left(O T_{i j}\right)\left(\sigma_{w}\right)$.

Corollary 11 For any $w \in S_{n}$, the operator of quantum multiplication by the Schubert class $\sigma_{w}$ commutes with the operator $O$.

Proof - Proposition 9 and quantum Monk's formula (7) imply that the operator of quantum multiplication by a two-dimensional Schubert class $\sigma_{s_{k}}$ commutes with the twisted cyclic shift operator $O$. By Remark 3, for any $w \in S_{n}$, the operator of quantum multiplication by $\sigma_{w}$ commutes with $O$.

This also proves Theorem 4 , because it is equivalent to Corollary 11 .

\section{Transition graph}

The Bruhat order $B r_{n}$ is the partial order on the set of all permutations in $S_{n}$ given by the following covering relation: $u \rightarrow w$ if $w=u s_{a b}$ and $\ell(w)=\ell(u)+1$. In other words, $u \rightarrow w$ if $\sigma_{w}$ appear in the expansion of $\sigma_{s_{k}} \cdot \sigma_{u}$ for some $1 \leq k<n$ (the product in the usual cohomology ring). 
The analogue of the Bruhat order for the quantum cohomology ring is the following transition graph. The transition graph $\operatorname{Tr}_{n}$ is the directed graph on the set of permutations in $S_{n}$. Two permutations are connected by an edge $u \rightarrow w$ in $T r_{n}$ if $w=u s_{a b}$ and either $\ell(w)=\ell(u)+1$ or $\ell(w)=\ell(u)-\ell\left(s_{a b}\right)$. We will label the edge $u \rightarrow u s_{a b}$ by the pair $(a, b)$. Equivalently, two permutations are connected by the edge $u \rightarrow w$ in $T r_{n}$ whenever $\sigma_{w}$ appear in the expansion of the quantum product $\sigma_{s_{k}} * \sigma_{u}$ for some $1 \leq k<n$.

Proposition 9 implies the cyclic symmetry of the transition graph:

Corollary 12 The transition graph $T r_{n}$ is invariant under the cyclic shift: $w \mapsto o w$, for $w \in S_{n}$.

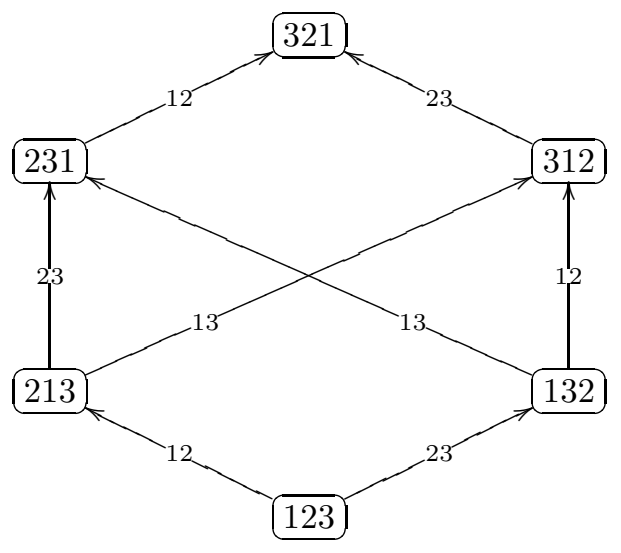

Figure 1: Bruhat order $\mathrm{Br}_{3}$.

Figures 1 and 2 show the Bruhat order $B r_{3}$ and the transition graph $\operatorname{Tr}_{3}$. The transition graph $T r_{3}$ is obtained by adding several new edges to $B r_{3}$, which makes the picture symmetric with respect to the cyclic group $\mathbb{Z} / 3 \mathbb{Z}$. The generator $o$ of the cyclic group rotates the graph $\operatorname{Tr}_{3}$ by $180^{\circ}$ clockwise. 


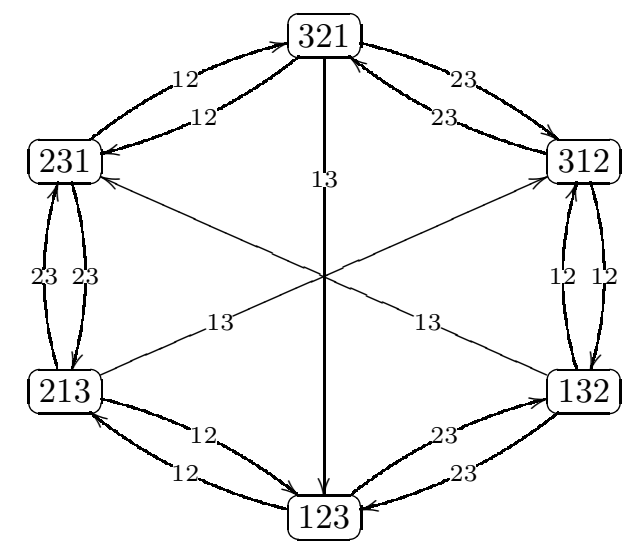

Figure 2: Transition graph $T r_{3}$.

\section{References}

[1] S. Agnihotri, C. Woodward: Eigenvalues of products of unitary matrices and quantum Schubert calculus, Math. Research Letters, 5 (1998), 817-836.

[2] N. Bergeron, F. Sottile: Schubert polynomials, the Bruhat order, and the geometry of flag manifolds, Duke Math. J. 95 (1998), no. 2, 373-423.

[3] S. Fomin, S. Gelfand, A. Postnikov: Quantum Schubert polynomials, J. Amer. Math. Soc. 10 (1997), 565-596.

[4] S. Fomin, A. Kirillov: Quadratic algebras, Dunkl elements, and Schubert calculus, Advances in Geometry, Progress in Mathematics 172, Birkhäuser, Boston, 1999, 147-182.

[5] W. Fulton, R. Pandharipande: Notes on stable maps and quantum cohomology, preprint alg-geom/9608011; also report no. 4, Institut Mittag-Leffler, 1996.

[6] A. Postnikov: On a quantum version of Pieri's formula, Advances in Geometry, Progress in Mathematics 172, Birkhäuser, Boston, 1999, 371-383. 